\title{
Kinematic signatures of triaxial stellar systems
}

\author{
RICHARD ARNOLD, TIM DE ZEEUW \\ Sterrewacht Leiden \\ and \\ CHRIS HUNTER \\ Sterrwacht Leiden and Florida State University
}

\begin{abstract}
Analytic dynamic models of triaxial stellar systems, stich as elliptical galaxies and galactic bulges, can be used to calculate the velocity fields of systems in a wide range of potentials without the need for orbit integrations. We present results from a firs $t$ application of these models, in the form of velocity fields projected onto the sky. The appearance of the velocity field depends strongly on the viewing angle. Thin orbit models provide a theoretical upper limit to streaming in all possible kinematic models in a given potential.
\end{abstract}

In order to model the observed velocity field of a stellar system, one needs to know the mass and momentum densities of the stellar orbits in the gravitational potential. In an arbitrary potential these densities can only be determined by direct numerical integration of the motion of a test particle around each orbit.

Such numerical integrations can be avoided in certain special potentials where the orbital densities are known analytically. Hunter and de Zeeuw (1991) have found analytic descriptions of self-gravitating stellar systems built from 'thin' orbits which have no radial thickness - in Stäckel potentials. In the cases where all stars rotate in the same sense, these thin orbit models represent the maximum streaming possible in the adopted potential. Any other model will consist of thicker orbits, or will have contra-rotating stellar populations, and will have less streaming.

We have investigated the maximum streaming models based on perfect ellipsoid potentials with a variety of axis ratios. We project the mass and momentum densities of the models onto the sky for a range of viewing angles, yielding in each case a two dimensional map of radial velocities. Two examples are shown in figure 1.

As can be seen from figure 1, and as has already been shown in detail by Statler (1991), the velocity field of a given triaxial stellar system can look radically different from different directions. For example, consider the difference in slope of the zero velocity curve (ZVC) between small and large radii in a velocity field. In figure 1(a) the difference in slope is very large $\left(135^{\circ}\right)$, and the velocity field shows a counterrotating core. Figure $1(\mathrm{~b})$, which is the same model viewed from a different direction, and which also has same projected ellipticity, shows a ZVC slope difference in slope of only $5^{\circ}$. Contour maps of ZVC slope difference as a function of viewing direction are shown in figure 2 for two different models. For a range of viewing angles - larger in model (a) than model (b) - a counter-rotating core is visible.

From the thin orbit models we learn for the first time how to put bounds on the streaming velocities in a triaxial potential. Using these bounds, and the further constraints provided by the equation of continuity, we create more realistic models with less streaming and thicker tube orbits. Because the mass and momentum densities have an analytic form, the resulting models provide a unique means to survey a wider range of galaxy shapes than would be feasible with methods relying on numerical orbit integration. 

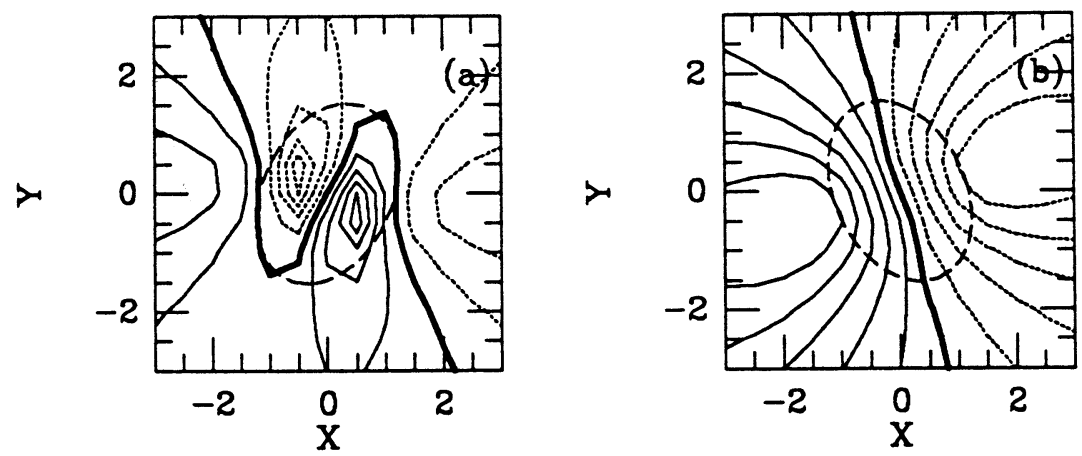

Fig. 1. Two views of the same prolate model $(b / a=0.5, c / a=0.375)$. The heavy curve is the zero velocity curve: the locus of points of zero radial velocity. The ellipse is the projection of the ellipsoid which encloses half of the mass, and indicates the shape of the light distribution. [Viewing angles $(\theta, \phi):(\mathrm{a})\left(45^{\circ}, 15^{\circ}\right),(\mathrm{b})\left(45^{\circ}, 165^{\circ}\right)$ ]
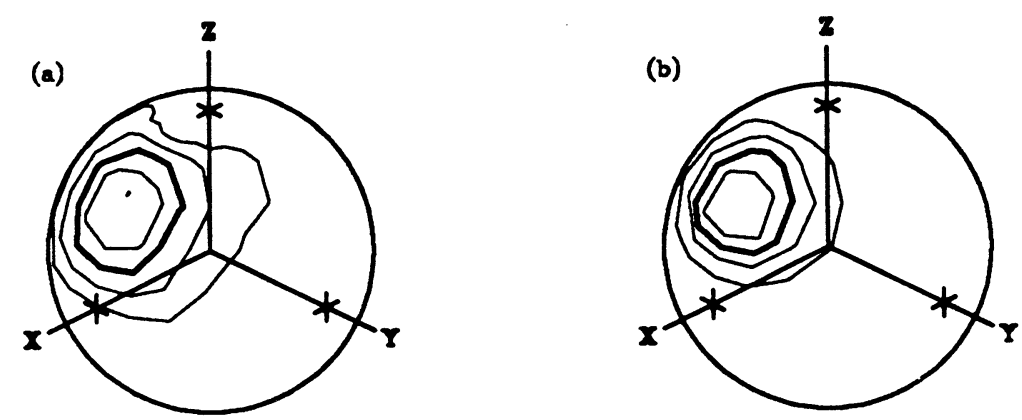

Fig. 2. The difference in slope of the zero velocity contour between small and large radii in the velocity field, drawn on the unit sphere of viewing angles. The results for two models are shown: (a) $b / a=0.5, c / a=0.375$; (b) $b / a=0.75, c / a=0.625$; The contours are spaced at intervals of $30^{\circ}$. For viewing directions inside the heavy contour the deviation is greater than $90^{\circ}$ and the velocity field shows a distinct counterrotating core.

It is our intention to explore the probability distributions of the observables which can be derived from velocity fields (ellipticity, minor/major axis rotation etc.) in a variety of potentials using the thin orbit models. The ultimate aim is to constrain the possible shapes of stellar systems from the observed distributions (see for example Franx, Illingworth and de Zeeuw 1991).

\section{Acknowledgements}

CH acknowledges support from the NSF, and a visitor's grant in the Netherlands from NWO. RA is supported by an ESA external fellowship.

\section{References}

Franx, M., nlingworth, G. D., \& de Zeeuw, P. T., 1991, Astrophys. J., 383112

Hunter, C., \& de Zeeuw, P. T., 1991, Astrophys. J., 38979

Statler, T. S., 1991, Astron. J., 102882 